\title{
Learning From Number Board Games: You Learn What You Encode
}

\author{
Elida V. Laski \\ Boston College
}

\author{
Robert S. Siegler \\ Carnegie Mellon University
}

\begin{abstract}
We tested the hypothesis that encoding the numerical-spatial relations in a number board game is a key process in promoting learning from playing such games. Experiment 1 used a microgenetic design to examine the effects on learning of the type of counting procedure that children use. As predicted, having kindergartners count-on from their current number on the board while playing a $0-100$ number board game facilitated their encoding of the numerical-spatial relations on the game board and improved their number line estimates, numeral identification, and count-on skill. Playing the same game using the standard count-from-1 procedure led to considerably less learning. Experiment 2 demonstrated that comparable improvement in number line estimation does not occur with practice encoding the numerals 1-100 outside of the context of a number board game. The general importance of aligning learning activities and physical materials with desired mental representations is discussed.
\end{abstract}

Keywords: board games, encoding, numerical magnitude, early childhood mathematics, microgenetic

Ensuring that children acquire foundational numerical knowledge by the end of kindergarten is central to improving mathematics achievement in the United States. Kindergartners' mathematical knowledge predicts subsequent mathematics achievement test scores in elementary, middle, and even high school (Aunola, Leskinen, Lerkkanen, \& Nurmi, 2004; Duncan et al., 2007; Jordan, Kaplan, Ramineni, \& Locuniak, 2009; LeFevre et al., 2010; Stevenson \& Newman, 1986). The strength of the predictive relations between early understanding and later achievement is much greater in math than in other, less hierarchical, domains.

Precise representations of numerical magnitudes are crucial for effective mathematics learning. The degree to which children's estimates of numerical magnitude on a number line increase linearly with the size of the numbers being estimated correlates strongly with the children's mathematics achievement test scores at all grade levels from kindergarten through eighth grade (Booth \& Siegler, 2006; Geary, Hoard, Byrd-Craven, Nugent, \& Numtee, 2007; Holloway \& Ansari, 2009; Schneider, Grabner, \& Paetsch, 2009; Siegler \& Booth, 2004; Siegler, Thompson, \& Schneider, 2011). Moreover, more linear numerical magnitude representations in first grade are associated with faster growth in math skills

This article was published Online First October 7, 2013.

Elida V. Laski, Department of Counseling, Developmental, and Educational Psychology, Boston College; Robert S. Siegler, Department of Psychology, Carnegie Mellon University.

Thanks are due to the children, teachers, and principals of PROPEL Homestead and PROPEL East Schools and to the Department of Education (Grants R305H020060 and R305B040063) and the National Institutes of Health (Grant HD 19011) for supporting this research.

Correspondence concerning this article should be addressed to Elida V. Laski, Department of Counseling, Developmental, and Educational Psychology, Boston College, 140 Commonwealth Avenue, Chestnut Hill, MA 02467, or to Robert S. Siegler, Department of Psychology, Carnegie Mellon University, Pittsburgh, PA 15213. E-mail: laski@bc.edu or rs7k@andrew.cmu.edu over the elementary school years, even after controlling for alternative predictive factors, such as intelligence and working memory (Geary, 2011). Causal relations have also been established; experiences that improve the numerical magnitude knowledge of randomly selected children also improve their subsequent learning of arithmetic and other mathematical skills (Booth \& Siegler, 2008; Laski \& Siegler, 2007; Siegler \& Ramani, 2009; Whyte \& Bull, 2008).

In particular, playing linear number board games has been shown to improve preschoolers' knowledge of numerical magnitudes and a range of other numerical skills (Ramani \& Siegler, 2008; Siegler \& Ramani, 2008, 2009; Whyte \& Bull, 2008). How these board games produce learning is poorly understood, however. This is unfortunate, because identifying the mechanisms through which number board games exercise their effects could provide information about general learning principles, as well as how to optimize and extend the benefits of playing such games.

In the present study, we propose a theoretical framework-the cognitive alignment framework-for identifying how physical materials and mental and physical actions with those materials produce learning. Guided by this framework, we used a microgenetic design to test the hypothesis that a seemingly minor difference in the type of counting during game playing influences encoding of the numerical-spatial relations on the game board and thus contributes to learning. This approach allowed us to describe the process of change during game playing, to test a framework for designing instructional materials, and to explore the usefulness of developmental psychology methods for understanding educational interventions.

\section{The Cognitive Alignment Framework}

Although the use of physical materials and games in mathematics instruction is often advocated (Ainley, 1990; Ball, 1992), empirical support for their effectiveness is mixed (McNeil \& Jarvin, 2007). For instance, when Din and Calao (2001) provided kindergartners from low-income backgrounds with educational 
games aimed at improving mathematical knowledge, the children's mathematics abilities did not improve relative to peers who were not given the games.

The cognitive alignment approach provides a theoretical framework for considering how and when physical materials are most likely to produce effective learning. Its basic principle is this: The more precisely that physical materials and learning activities are aligned with the desired mental representation, the more likely students are to acquire that representation. One source of support for this framework comes from research on the development of the use of symbols and analogical reasoning. This research has suggested that physical materials that are closely aligned to the desired mental representation increase analogical transfer (Chen, 1996; DeLoache, Kolstad, \& Anderson, 1991; Gentner \& Markman, 1997; Goswami, 1996).

In another application of this principle, Siegler and Ramani (2009) reasoned that a linear board game would be more closely aligned with the desired linear mental representation of numerical magnitudes than would a circular board game and therefore would produce greater acquisition of the desired linear representation. Consistent with the cognitive alignment framework, the linear board game produced greater learning of numerical magnitudes, as measured by both number line estimation and numerical magnitude comparison.

Even with the most highly aligned learning materials, however, learning can fail. This possibility is especially likely in situations where children can interact with the materials in ways that do not require the desired encoding (Uttal, O'Doherty, Newland, Hand, \& DeLoache, 2009). Findings from numerous domains indicate that learners often fail to encode relevant dimensions, that inadequate encoding impairs learning, and that instructions that improve encoding of key features or relations improve learning (Alibali, 1999; Barrett, Abdi, Murphy, \& Gallagher, 1993; Blaxton, 1989; Brown, Kane, \& Echols, 1986; Chi, 1978; McCloskey \& Kaiser, 1984; Ornstein et al., 1998; Siegler, 1976; Siegler \& Chen, 1998; Staszewski, 1988). Thus, the cognitive alignment framework posits that there is a need, even with the best designed learning materials, for activities that direct learners' behaviors in ways that promote the encoding of the features relevant to the desired mental representation.

\section{Analysis of Number Board Games}

Siegler and Booth (2004) hypothesized that experience playing a linear number board game such as Chutes and Ladders contributes to knowledge of numerical magnitudes. Their reasoning was that such games provide visual, kinesthetic, auditory, and temporal cues to the linear structure of the number system. Imagine a board game similar to the first row of Chutes and Ladders, that is, with the numbers 1-10 arranged in equal-size squares progressing from left to right. Such a game would allow computation of correlations between numerals and the magnitudes they represent. For example, it takes twice as many physical movements and roughly twice as much time to move one's token from the origin to 6 as to 3 ; reaching 6 also requires the child to move the token twice as far from the origin and to say and hear twice as many number words.

Consistent with this analysis, experience playing a linear number board game that has a setup similar to the first row of Chutes and Ladders helps preschoolers from low-income backgrounds learn the magnitude of the numbers 0-10. Preschoolers who played such a game improved on two tasks that measure numerical magnitude knowledge-number line estimation and numerical magnitude comparison - as well as on counting, numeral identification, and ability to learn the answers to arithmetic problems (Ramani \& Siegler, 2008; Siegler \& Ramani, 2008, 2009; Whyte $\&$ Bull, 2008). In contrast, peers who engaged in other numerical activities (e.g., counting and identifying numerals) improved on those tasks but not on knowledge of numerical magnitudes (Siegler \& Ramani, 2009; Whyte \& Bull, 2008). The gains of children who played the linear number board game endured for at least 2 months (Ramani \& Siegler, 2008), and playing the game helped children from middle-income as well as low-income families (Ramani \& Siegler, 2011).

One feature of the number board game that differentiated it from the way that such games are usually played was that children were required to say the names of the numbers in the squares as they moved their token on each turn. Thus, children who were on the square that contained the number 5 and who spun a 2 were required to count-on by saying " $6,7$. ." If they were unable to count-on from a given number, the experimenter modeled the correct procedure and then asked the child to repeat it until the child executed it correctly. This procedure differs from the typical procedure of counting-from-1 the number of spaces indicated on the spinner or dice regardless of the token's initial position (e.g., children on 5 who spin a 2 typically say, "1, 2").

Whether children count-on from the number in the square of their current position on the game board or count-from-1 might seem like a minor procedural detail, but the cognitive alignment framework suggested that it is crucial to the effects of playing the number board game. Children can easily play number board games without encoding the numbers on the game board or their associated magnitudes; they can spin the spinner, count-from-1 to whatever number they spun, and ignore the numbers in the squares.

It is uncertain when children begin to automatically activate numerical magnitude representations when seeing Arabic numerals between 0 and 9 . Results of some studies have suggested that children may do so by the age of 7 years (Bugden \& Ansari, 2011); results of others have suggested it is not until age 8 or 9 (Berch, Foley, Hill, \& McDonough-Ryan, 1999; Girelli, Lucangeli, \& Butterworth, 2000; van Galen \& Reitsma, 2008). In either case, the findings have suggested that kindergartners, who generally are 5or 6-year-olds, are unlikely to automatically encode the magnitude of all the numerals through 100. Thus, maximizing the likelihood of acquiring a linear representation requires learning activities that promote encoding of the numbers on the board, so that children can connect these numbers to the magnitude cues that accompany them (e.g., the time required to reach each number).

\section{The Present Study}

The present study had three main goals. One was to explore the generality of the benefits of playing numerical board games: Would activities that produced substantial gains in knowledge of numbers in the $0-10$ range produce similar gains for numbers in the $0-100$ range? This latter range is more challenging not only because it includes 10 times as many numbers but also because logistical constraints make it impractical to use a strictly linear $1 \times$ 100 game board. Forming a linear representation from experience 
with a semilinear board, such as the $10 \times 10$ matrix used in Chutes and Ladders, requires learners to integrate their knowledge across the rows of the board, which might be difficult or impossible for children as young as the kindergartners who participated in the present study. As noted previously, nonlinearly (circularly) organized boards produced little learning of numerical magnitudes in previous studies (Ramani \& Siegler, 2011; Siegler \& Ramani, 2009). On the other hand, the $10 \times 10$ game board has the advantage of embodying the base- 10 system in a transparent way, with the rows representing decades and the columns representing units (e.g., in the present board, 47 was located at the fourth row, seventh column). The present study tested the generality of the board game's effects not only for improving knowledge of a larger range of numbers but also for improving counting-on skill and number identification.

The second goal of the study was to identify specific aspects of the number board game that promote learning. In particular, we hypothesized that counting-on while moving the token promotes greater encoding of numerals and their positions on the game board than counting-from-1. This greater encoding, in turn, was predicted to increase the extent to which children extracted the correlations between the number and the several cues to magnitude associated with reaching that number and thus to increase the linearity of their representation of numerical magnitudes. This hypothesis was tested in Experiment 1 by manipulating the type of counting during game playing-children either counted-on from the number in the square of their current position on the game board or counted-from-1 the number of spaces moved. Experiment 2 tested the benefit of encoding numerals outside of a numerical board game context to separate out the benefit of encoding numerals while counting sequences of them from the benefit of engaging in the same process in the context of a number board game with a semilinear spatial layout.

The third goal of the study was to examine how changes in numerical knowledge occur while playing board games. In Experiment 1 , a microgenetic design was used to address this goal. The central feature of microgenetic designs is frequent assessment of knowledge and cognitive processes during the period in which learning is occurring (Siegler, 2006). Such a design makes it possible to examine how changes in cognitive processes, in this case encoding, are related to changes in the desired knowledge, in this case linear representations of numerical magnitudes. Experiment 1 included a pretest session, four game playing sessions, and a posttest session; in each of the game-playing sessions, children played the $0-100$ board game twice. Encoding was measured after the first and third game-playing sessions (the second and fourth sessions overall); numerical magnitude representations were mea- sured after the second and fourth game-playing sessions (the third and fifth sessions overall). This microgenetic design allowed examination of the process as well as the products of change.

\section{Experiment 1}

\section{Method}

Participants. Of the 42 kindergartners who participated (mean age $=5.8$ years), $42 \%$ were African American, $52 \%$ Caucasian, and $7 \%$ Other. The children were recruited from two charter schools serving low- to lower-middle-income families; the percentages of children eligible for the free or reduced-fee lunch program in the two schools were $93 \%$ and $55 \%$, respectively.

Children within each classroom at each school were randomly assigned to one of two conditions: count-from- 1 or count-on. The count-from- 1 condition included 21 children (mean age $=5.80$, $33 \%$ female, $33 \%$ African American, 52\% Caucasian, and $15 \%$ Other). The count-on condition also included 21 children (mean age $=5.80,52 \%$ female, $48 \%$ African American, 52\% Caucasian, and $0 \%$ Other). An additional three children (one in the countfrom-one condition and two in the count-on condition) were presented the pretest but did not complete the experiment because of extended absences or refusal to participate.

All children were tested in the spring of their kindergarten year by the same experimenter. Testing of the children in the two conditions was interleaved, so that children in them were matched for the time of year of their participation.

Design. Children met individually with an experimenter for two sessions per week for 3 weeks. As Table 1 illustrates, in Sessions 1 and 6, children completed the pretest and posttest; in Sessions 2-5, they played the board game.

Pretest/posttest sessions (Sessions 1 and 6). In these sessions, children were presented five tasks designed to measure numerical knowledge and encoding of the game board. On the number line estimation task, participants were read a numeral and asked to mark its position on a number line that included labeled endpoints ( 0 and 100) but no other markings. Previous estimates were not visible on later trials. Following two practice trials on which children were asked to indicate the positions of 0 and 100 and shown their location if needed, children were presented 22 test trials without feedback. The numbers presented were 2, 3, 5, 8, 12 , $17,21,26,34,39,42,46,54,58,61,67,73,78,82,89,92$, and 97. A different random order of the numbers was generated for each child at pretest and posttest. The interitem reliability, based on children's percentage of absolute error (PAE) on each trial, was $\alpha=.82$ at the first administration.

Table 1

Experiment 1: Constructs, Measures, and Time Points of Measurement

\begin{tabular}{|c|c|c|c|c|c|c|}
\hline \multirow[b]{2}{*}{ Construct and measure } & \multirow{2}{*}{$\frac{\text { Pretest }}{\text { Session } 1}$} & \multicolumn{4}{|c|}{ Board game experience } & \multirow{2}{*}{$\frac{\text { Posttest }}{\text { Session } 6}$} \\
\hline & & Session 2 & Session 3 & Session 4 & Session 5 & \\
\hline Number line estimation & $X$ & & $\mathrm{X}$ & & $\mathrm{X}$ & $\mathrm{X}$ \\
\hline Numeral identification & $\mathrm{X}$ & & & & & $\mathrm{X}$ \\
\hline Count-on & $\mathrm{X}$ & & & & & $X$ \\
\hline Game board reproduction & $\mathrm{X}$ & & & & & $\mathrm{X}$ \\
\hline Numeral position encoding & $\mathrm{X}$ & $\mathrm{X}$ & & $\mathrm{X}$ & & $\mathrm{X}$ \\
\hline
\end{tabular}


On the numeral identification task, children were presented 28 numerals between 0 and 100 on a computer screen and asked to name them. The numerals were $1,2,3,4,5,6,7,8,9,13,15,24$, $27,31,33,40,43,51,57,64,66,72,77,85,86,90,95$, and 100 . The interitem reliability was $\alpha=.93$ at the first administration.

On the counting-on task, children were asked to "start counting with $a$ and count up $N$ more numbers" with $a$ being 7, 18, 37, or 84 and $N$ being 3,5 , or 8 . The 12 trials were presented in one of two counterbalanced, semirandom orders (random except for the stipulation that the same starting point was not used on consecutive trials). The interitem reliability was $\alpha=.85$ at the first administration.

The game board reproduction task provided a measure of encoding of the base-10 structure of the board. Children were shown the board for $30 \mathrm{~s}$ and then were given $5 \mathrm{~min}$ to draw it on a tablet PC. At the outset, children were told that they should look carefully at the board and notice what was really important, because they were going to draw it afterward. Because limited fine motor skill and ability to write numerals could interfere with the intended depiction, children were asked to explain what they intended in their drawings and to describe what else they might include if they had more time. The final drawings and videotaped explanations were coded according to whether children drew or described 12 features of the game board relevant to its base-10 organization: gridlike pattern, $10 \times 10$ grid, numbers arranged in rows, at least one row with 10 boxes or with labeling that stopped at a decade, two or more rows with 10 boxes or labeling that stopped at a decade, numbers increasing from bottom to top, numbers increasing from left to right, 0 labeled, 100 labeled, 0 labeled in correct position (bottom left corner), 100 labeled in correct position (top right corner), and some numerals between 0 and 100 written. Children received 1 point for each feature included. A subset (10\%) of the drawings was scored by a second rater. The scoring of the drawings was highly reliable: Experiment $1 \kappa=.85, p<$ .01 , and Experiment $2 \kappa=.87, p<.01$.

On the numeral position encoding task, children were presented a computer screen with a $10 \times 10$ matrix, with all squares in the matrix being blank, except for 0 and 100, which were labeled with those numerals. Before the first trial, children were allowed to look at the physical game board for $30 \mathrm{~s}$. Then they were asked to move their cursor on the computer screen to the square where a number at the top of the screen on that trial belonged and to click their mouse when the cursor was on it. Immediately after their response, a new number appeared. In all, 23 numbers were presented in random order: $5,8,10,12,15,16,21,28,31,39,40,46,51,54$, $65,67,73,75,77,82,85,90$, and 92 . The interitem reliability was $\alpha=.68$ at the first administration.

Game-playing sessions (Sessions 2-5). In each of these sessions, children twice played the board game Race to Space with the experimenter. All children used a game board on which the numbers $1-100$ were arranged in a $10 \times 10$ matrix (see Figure 1 ). The blue background color of the board deepened every two rows, providing an added cue to numerical magnitude. The spinner, which determined how far participants would move their tokens on each turn, had five sections labeled 1-5.

Children participated in one of two experimental conditions that differed only in the type of counting required as children moved their token. In the count-from-1 condition, children counted aloud from 1 as they moved their token until they reached the number

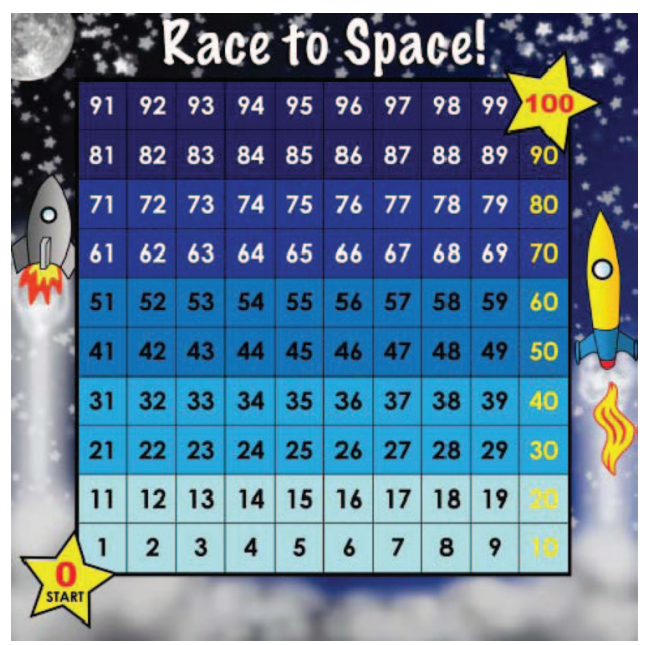

Figure 1. Number board game used with children in both conditions of Experiment 1 and in Experiment 2.

indicated on the spinner (e.g., children on 17 who spun a 2 said "1" as they put their token on the square labeled 18 and "2" as they put their token on the square labeled 19). In the count-on condition, children counted-on from the number on the square where they began the turn (e.g., children on 17 who spun a 2 said "18, 19"). In both conditions, if the child could not perform the requested activities, the experimenter helped with the counting and with moving the token the appropriate number of squares. To equalize exposure to the counting sequence and the support provided, kindergartners in the count-from- 1 condition were asked to count aloud to 100 before playing each game and were helped whenever they made a counting error or were unable to produce the next number in the counting sequence.

In both conditions, the experimenter introduced the game by saying that its purpose was to help children learn more about the numbers between 0 and 100 . Once in each 10 numbers, the experimenter drew children's attention to their position on the game board (e.g., "Look! You're on 22"). Other incidental comments were kept to a minimum and were made only when the child asked a question or made a comment (e.g., "I'm winning").

No set time was imposed on the time of the training sessions; the training sessions went as long as was needed for the child to play the board game to completion twice. Playing each game to completion (i.e., until both players reached 100) ensured that children were exposed to the entire range of numbers. The mean time of the four training sessions was $19.36 \min (S D=1.90)$ in the count-from-1 condition and $25.28 \mathrm{~min}(S D=5.85)$ in the count-on condition, thus necessitating statistical controls for time on task.

\section{Results}

Preliminary analyses found no gender, classroom, or school effects. Thus, these variables were not considered in subsequent analyses. Because the count-on condition led to longer gameplaying sessions, the average time of each child's training sessions 
was used as a covariate in the analyses of variance and regression analyses.

\section{Effects of experimental condition.}

Multivariate analyses. The first analysis examined the effects of type of counting on the five outcome measures. For the counton, numeral identification, and numeral position encoding tasks, the dependent measure was the percentage of correct answers. For the game board reproduction task, the dependent measure was the number of relevant features included in children's drawings or cited by the children when explaining their intention. For the number line estimation task, the dependent measure was a composite score calculated by summing individuals' $z$ scores for the percentage of absolute error $=($ lestimate-estimated quantityl/scale of estimates $) \times 100$, linearity, and slope of the best fitting linear function.

A 2 (condition: count-from- 1 or count-on) $\times 2$ (session: pretest or posttest) repeated-measures multivariate analysis of variance was conducted on the five measures, controlling for the average time of individuals' training sessions. Effects emerged for session, $F(5,34)=3.76, p<.01, \eta_{\mathrm{p}}^{2}=.36$, and for the condition by session interaction, $F(5,34)=5.54, p<.01, \eta_{\mathrm{p}}^{2}=.45$.

To better understand the interaction, and to examine the consistency of results across tasks, repeated-measures analyses of variance (ANCOVAs) were conducted for each task. Consistent with the hypothesis that counting-on would produce greater learning than counting-from-1, a condition by session interaction was found for all tasks, except for numeral identification, on which both groups performed near ceiling at pretest and posttest (see Figure 2).

Number line estimation. A repeated-measures ANCOVA on the number line estimation composite score indicated that children's number line estimates varied with the condition by session interaction, $F(1,38)=7.72, p<.01, \eta_{\mathrm{p}}^{2}=.17$. To better understand this interaction, $t$ tests were used to examine differences between conditions at pretest and posttest for each measure of number line estimation: percentage of absolute error, linearity, and slope. Condition affected improvement on each indicator. As shown in Figure 2a, the count-from-1 condition produced no pretest-posttest change in number line PAE: $21 \%(S D=7 \%)$ at pretest and $20 \%(S D=7 \%)$ at posttest. In contrast, the count-on condition produced pretest-posttest improvement, 20\% $(S D=$ $7 \%)$ at pretest versus $14 \%(S D=7 \%)$ at posttest, $t(20)=5.60$, $p<.0005, d=0.86$. The two conditions did not differ at pretest ( $21 \%$ and $20 \%$, respectively) but did differ at posttest: $19 \%$ versus $14 \%$, respectively, $t(40)=2.20, p=.03, d=0.71$.

The count-from- 1 condition also produced no pretest-posttest improvement in the linearity of number line estimates (see Figure $2 b$ ). Mean percentage of variance in individual children's estimates that was accounted for by the best fitting linear function was $46 \%$ ( $S D=$ $23 \%)$ at pretest and $47 \%(S D=29 \%)$ at posttest. In contrast, the count-on condition produced substantial pretest-posttest improvement in the linearity of individual children's estimates, $49 \%$ (SD = $29 \%)$ at pretest versus $69 \%$ at posttest $(S D=22 \%), t(20)=4.53$, $p<.0005, d=0.77$. The two conditions did not differ at pretest $(46 \%$ and $49 \%$, respectively) but did differ at posttest, $47 \%$ versus $69 \%$, respectively, $t(40)=2.80, p<.01, d=0.87$.

The count-from-1 condition again produced no pretest-posttest improvement in the mean slopes of individual children's number line estimates, $.54(S D=.20)$ at pretest and $.48(S D=.24)$ at posttest (see Figure 2c). In contrast, the count-on condition produced substantial pretest-posttest improvement, .47 $(S D=.25)$ versus $.67(S D=.17), t(20)=4.81, p<.0005, d=0.93$. The mean slopes of number line estimates of children in the two conditions did not differ at pretest (.54 and .47 , respectively) but differed at posttest, .48 versus .67 , respectively, $t(40)=2.94, p<$ $.01, d=0.91$.

Numeral identification. Percentage of correct numeral identifications varied with session, $F(1,38)=10.5, p<.01, \eta_{\mathrm{p}}^{2}=.22$, but not with the session by condition interaction.

Count-on. Number of correct count-on sequences varied with the condition by session interaction, $F(1,38)=13.55, p<.01$, $\eta_{\mathrm{p}}^{2}=.26$. Count-on accuracy of children in the count-from-1 condition did not improve: $23 \%(S D=25.30 \%)$ on pretest and $23 \%(S D=26.82 \%)$ on posttest (see Figure 2e). In contrast, accuracy of children in the count-on condition improved from pretest to posttest from $25 \%(S D=22.89 \%)$ to $54 \%$ ( $S D=$ $29.54 \%), t(20)=4.37, p<.0005, d=1.10$. The two conditions did not differ at pretest (23\% and $25 \%$, respectively) but did differ at posttest, $23 \%$ versus $54 \%$, respectively, $t(40)=3.56, p<.01$, $d=1.10$.

Numeral position encoding. Correct locations of the position of numerals in the matrix varied with session, $F(1,38)=10.81$, $p<.01, \eta_{\mathrm{p}}^{2}=.22$, and with the condition by session interaction, $F(1,38)=12.61, p<.01, \eta_{\mathrm{p}}^{2}=.25$. Children in the count-from- 1 condition improved from $16 \%(S D=13.13 \%)$ correct locations of numerals on the pretest to $28 \%(S D=20.05 \%)$ on the posttest, $t(20)=4.16, p<.0005, d=0.71$. Children in the count-on condition, however, made larger improvements, from $17 \%$ ( $S D=$ $13.23 \%)$ to $44 \%(S D=22.53 \%), t(20)=6.79, p<.0005, d=$ 1.46 (see Figure 2f). The two conditions did not differ at pretest (16\% and $17 \%$, respectively) but did differ at posttest, $28 \%$ versus $44 \%$, respectively, $t(40)=2.42, p<.05, d=0.75$.

Game board reproduction. Accuracy of encoding of the relevant features and base-10 organization of the game board varied with the condition by session interaction, $F(1,38)=4.98, p=.03$, $\eta_{\mathrm{p}}^{2}=.12$ (see Figure $2 \mathrm{~g}$ ). The mean of the 12 relevant features cited by children in the count-from- 1 condition did not change significantly: $7.62(S D=2.78)$ on the pretest and $8.38(S D=$ 2.18 ) on the posttest. In contrast, children in the count-on condition improved from naming a mean of $6.76(S D=2.79)$ features on the pretest to naming a mean of $9.48(S D=2.68)$ on the posttest, $t(20)=7.41, p<.0005, d=1.00$. The number of features named by children in the two conditions did not differ significantly on either pretest (7.62 and 6.76, respectively) or posttest (8.38 and 9.48 , respectively), though the increase from pretest to posttest was larger in the count-on condition (2.72 versus .76 features, respectively).

Rate of learning. The microgenetic design allowed us to examine changes in number line estimation and encoding of the positions of the numerals on the board during training. Changes in the two conditions are shown in Figure 3.

Number line estimation. In the count-from- 1 condition, there was no significant improvement in PAE, linearity, or slope of number line estimates between any of the assessments. PAE was $21 \%(S D=7 \%)$ at pretest, $20 \%(S D=8 \%)$ at the end of the second training session, and $19 \%(S D=7 \%)$ at both the end of the fourth training session and at posttest. The amount of variance in each child's estimates accounted for by the linear function did not 
- Count-on

(a) Number Line Error

(b) Number Line Linearity
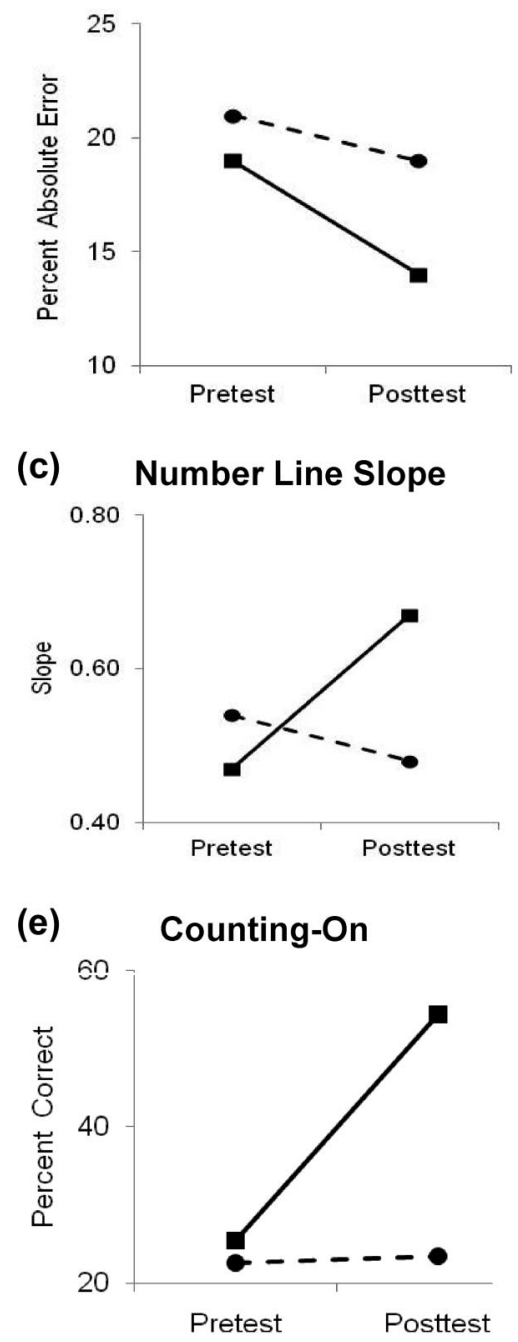

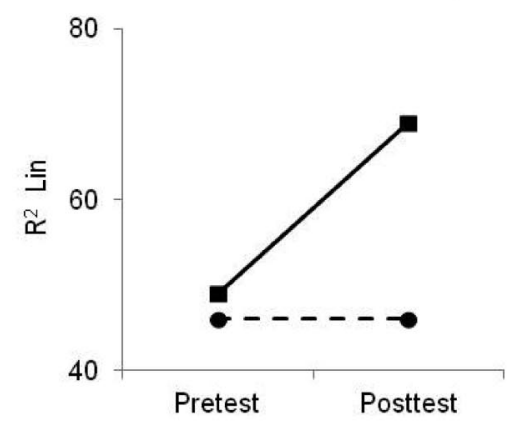

(d)

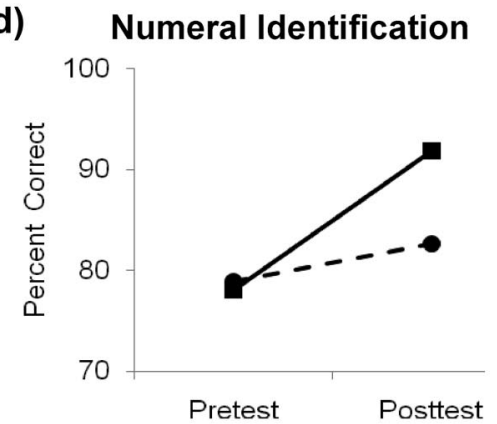

(f)
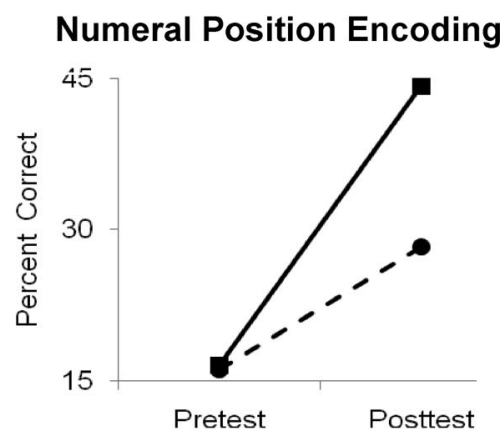

(g) Game Board Reproduction

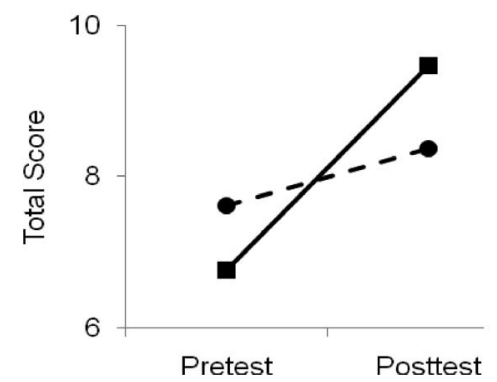

Figure 2. Experiment 1: Pretest and posttest performance of children in each condition for each outcome measure. Lin $=$ linear function.

change over the course of training; it averaged $46 \%$ (with a $S D$ ranging from $23 \%$ to $30 \%$ at the four points of measurement). The slope of the best fitting linear function also changed little from pretest $.54(S D=.20)$ to the end of the second training session, .49
$(S D=.19)$ to the fourth training session, and $.50(S D=.23)$ to the posttest $.48(S D=.24)$.

In contrast, in the count-on condition, there was more rapid and substantial improvement, particularly at the beginning of 
(a)

Number Line Error

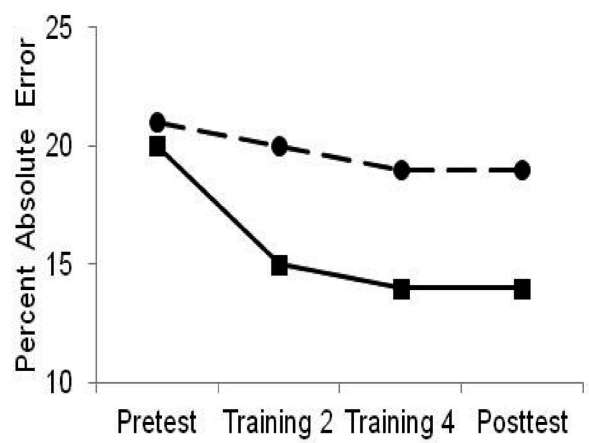

(c)

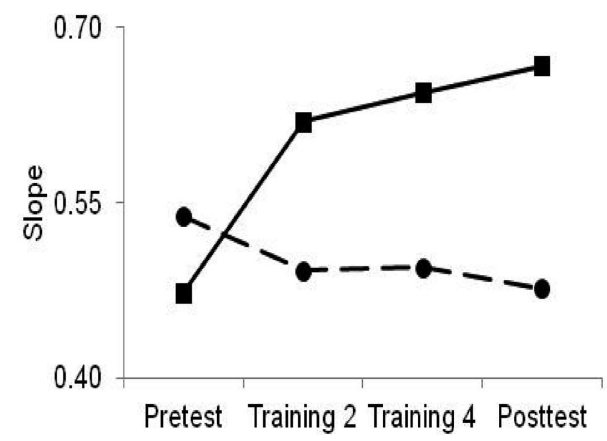

(b)

\author{
Number Line Linearity
}

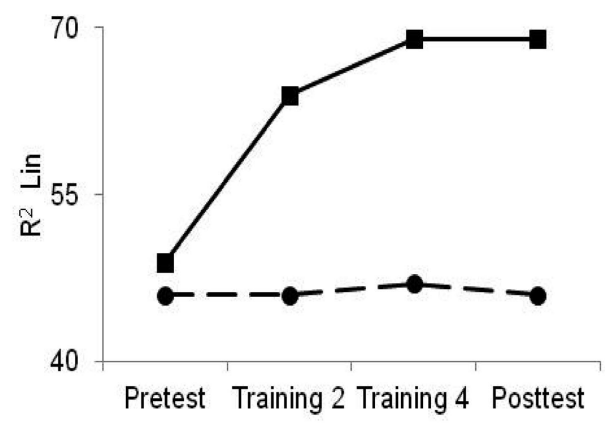

(d) Numeral Position Encoding

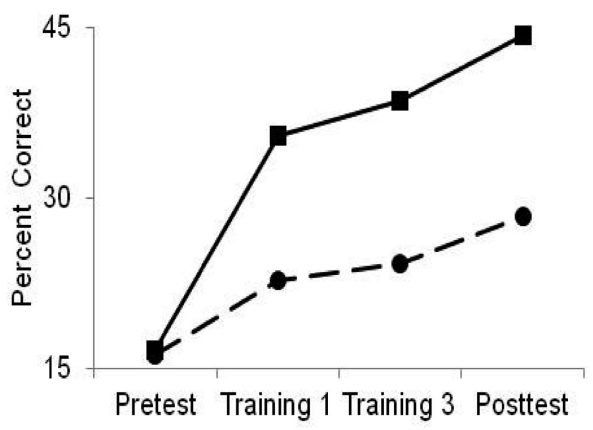

Figure 3. Experiment 1: Rate of improvement in number line estimation and encoding of numeral positions across training for each condition. Lin $=$ linear function.

training. Mean PAE of number line estimates decreased substantially at the beginning of training, from $20 \%(S D=7 \%)$ at pretest to $15 \%(S D=7 \%)$ at the end of the second training session, $t(20)=4.13, p<.01, d=0.71$. No further changes occurred in the fourth training session and posttest, both PAEs $=14 \%(S D=7 \%)$.

The same pattern of improvement was found for linearity and slope. Mean percentage of variance in individual children's estimates that was accounted for by the best fitting linear function increased substantially at the beginning of training, from $49 \%$ $(S D=29 \%)$ at pretest to $64 \%(S D=28 \%)$ at the end of the second training session, $t(20)=3.04, p<.01, d=0.58$. There was no significant change in the linearity of children's estimates between the ends of the second and fourth training sessions, 64\% and 69\% respectively ( $S D=25 \%$ ), nor from the end of the fourth training session to the posttest, both $=69 \%(S D=22 \%)$. Similarly, mean slope of individual children's number line estimates increased from pretest to the end of the second training session, .47 ( $S D=$ $.25)$ to $.62(S D=.19), t(20)=3.64, p<.01, d=0.68$, but did not change between the second and fourth training sessions, .62 and .64 , respectively $(S D=.17)$, nor between the fourth training session and the posttest, .64 and .67 , respectively $(S D=.17)$.

Numeral position encoding. In the count-from-1 condition, children's encoding of the position of the numerals on the game board improved from pretest to the end of the first training session, from $16 \%(S D=13.13 \%)$ to $23 \%(S D=16.15 \%), t(20)=2.43$, $p<.05, d=0.45$. There was no further significant change in the percentage of correct locations between the ends of the first and third training sessions, $23 \%$ and $24 \%$, respectively $(S D=$ $14.97 \%$ ), nor between the end of the third training session and the posttest, $24 \%$ and $28 \%$, respectively $(S D=20.04 \%)$. Learning in the count-on condition was similar but with two differences. One was that learning was greater at the beginning of training. Between pretest and the end of the first training session, percentage of correct locations of numerals more than doubled, increasing from $17 \%(S D=13.23 \%)$ to $35 \%$, respectively $(S D=21.27 \%), t(20)=$ $4.51, p<.0005, d=1.02$. The second difference was that correct identifications of numbers' locations increased between the end of the first training session and the posttest, $35 \%$ to $44 \%$, respectively $(S D=22.53 \%), t(20)=4.35, p<.0005, d=0.41$.

Encoding as a mediator. A simple mediation analysis was conducted, using the causal steps strategy (Baron \& Kenny, 1986) to test the hypothesis that improvement in encoding mediated acquisition of more linear and accurate number line estimates. We controlled for the mean time of training sessions, as well as pretest performance in number line estimation, numeral position encoding, and numeral identification. Pretest performance in counting from a number other than 1 on was not included as a covariate 
because it was not related to improvement in number line estimation in either condition. Numeral position encoding was used as the measure of encoding because it was the measure most closely related to our ideas about encoding spatial-numerical relations.

The regression analyses indicated that (a) experimental condition was related to posttest numeral position encoding accuracy ( $\beta=.43, p<.01)$, (b) experimental condition was related to posttest number line estimation $(\beta=.50, p<.01)$, and (c) posttest numeral position encoding accuracy was related to posttest number line estimation $(\beta=.57, p<.01)$. The relation between condition and posttest number line estimation decreased and was no longer significant when both condition and posttest numeral position encoding accuracy were included in a single model predicting posttest number line estimation $(\beta=.32, p=.09)$. The Sobel test $z$ value was $2.15, p=.03$.

We then used the bootstrapping method with bias-corrected confidence estimates to determine the indirect effects (Preacher \& Hayes, 2008). The product of the coefficients $(a b)$ for the indirect path from condition to posttest number line linearity through posttest numeral position encoding accuracy, controlling for initial performance on both tasks and mean time of training sessions, was significant (bias-corrected confidence interval $=0.08$ to 1.41 ). Thus, both the Sobel test and the bootstrapping method indicated that improved encoding of the numerical-spatial relations on the game board mediated the beneficial effects of the count-on condition.

\section{Discussion}

The results were consistent with the hypothesis that counting-on while moving the token in a numerical board game promotes greater encoding of numerals' spatial positions and, thus, greater learning about the numerical magnitudes of numbers. Counting-on led to improvements in number line estimation and encoding of the structure of the game board that were roughly twice as great as those made by children who counted-from-1 while playing the game.

However, there were two potentially important differences between the conditions other than the intended theoretical contrast. One was that children in the count-on condition had practice counting-on from numbers other than 1; counting-on per se, rather than counting-on in the context of the number board game, could have produced the gains observed in the experiment. The other potentially important difference was that children in the count-on condition could encode the printed Arabic numerals as they counted to 100 , whereas children in the count-from- 1 condition did so in the absence of printed numerals. Thus, it was unclear whether it was practice encoding numerals within the structure of a number board game or one or both of these other factors that benefited children's numerical magnitude knowledge.

Experiment 2 was designed to test whether practice counting-on and encoding printed numerals outside the context of a number board game would generate improvement in numerical magnitude knowledge comparable to that produced by playing the number board game using the count-on procedure. We expected that practice encoding numerals outside of the game context would produce improvement in numeral identification and counting-on similar to those shown by children in the count-on condition in Experiment 1 , because the experience relevant to those two skills would be highly similar or identical. However, it also was expected that this practice outside the board game context would not produce comparable improvement in number line estimation and encoding, because that experience would not produce the correlations between numerical magnitudes and kinesthetic, temporal, auditory, and visual cues that are present in the board game context.

\section{Experiment 2}

\section{Method}

Participants. Experiment 2 included 21 kindergartners (mean age $=5.9$ years) of whom 57\% were African American, 29\% Caucasian, and $14 \%$ Other. All of the children were recruited from the same two charter schools as in Experiment 1. As in Experiment 1 , all children were tested in the spring of their kindergarten year by the same experimenter.

Design. The design was the same as that in Experiment 1, but children did not complete the number line estimation and the numeral encoding tasks during Sessions 2-5.

Pretest/posttest sessions (Sessions 1 and 6). Children in Experiment 2 completed the same five tasks of numerical knowledge and encoding of the game board used in Experiment 1: number line estimation, numeral identification, counting-on, game board reproduction, and numeral position encoding.

Game-playing sessions (Sessions 2-5). The Experiment 2 game-playing sessions were similar to those in the count-from-1 condition in Experiment 1 but included an additional activity. After playing Race to Space as in the count-from-1 condition in Experiment 1, children named all of the Arabic numerals between 0 and 100 in order. The numerals were printed on individual cards and randomly divided into 34 sequences, varying between one and five numbers in length (a different set of 34 number sequences was used after each game). Summing across the cards in the 34 sequences, the children each counted from 1 to 100 in order with the printed numbers visible, as in the board game, but without the board being present. Except when presented the first sequence, children were required to count up from a number other than 1. For example, if the first sequence was 1,2 , then the next sequence was $3,4,5,6$. Children needed to read the numbers in the sequence and were corrected if they erred until they read them correctly. The experimenter and the children each took a turn naming the numerals in each sequence before starting the next sequence. The mean time of the four training sessions in Experiment 2 (28.91 min, $S D=5.39)$ was very similar to that in the count-on condition in Experiment 1 but slightly longer.

\section{Results}

Paired-samples $t$ tests were used to examine differences in performance from pretest to posttest. All dependent measures were the same as in Experiment 1.

Number line estimation. Unlike the children in the count-on condition in Experiment 1, children in Experiment 2 demonstrated no significant pretest-posttest improvement on any of the measures of number line estimation. Number line PAE was $16 \%$ $(S D=9 \%)$ at pretest and $14 \%(S D=6 \%)$ at posttest, $t(20)=2.02$, $p=.06, d=0.30$. Mean percentage of variance in individual children's estimates that was accounted for by the best fitting 
linear function was 58\% $(S D=29 \%)$ at pretest and $61 \%(S D=$ $24 \%$ ) at posttest, $t(20)=0.84, p=.41, d=0.15$. The mean slopes of individual children's number line estimates was $.56(S D=.26)$ at pretest and $.61(S D=.18)$ at posttest, $t(20)=1.22, p=.24, d=$ 0.22 .

Numeral identification and count-on. As expected, children did demonstrate pretest-posttest improvement on numeral identification and count-on. Accuracy of correct numeral identifications improved from $82 \%(S D=18.76 \%)$ at pretest to $90 \%(S D=$ $13.94 \%)$ at posttest, $t(20)=3.18, p<.01, d=0.48$. Counting-on accuracy improved from $18 \%$ at pretest $(S D=13.45 \%)$ to $24 \%$ at posttest $(S D=14.59 \%), t(20)=2.14, p=.05, d=0.45$.

Encoding. Children's improvement on the numeral position encoding task was comparable to that of children in the countfrom-one condition in Experiment 1: from 17\% $(S D=11.47 \%$ ) correct locations of numerals on the pretest to $26 \%(S D=13.32 \%)$ on the posttest, $t(20)=4.48, p<.0005, d=0.73$. Similarly, like children in the count-from- 1 condition in Experiment 1 and unlike those in the count-on condition of Experiment 1, children in Experiment 2 demonstrated no significant improvement in accuracy of encoding of the relevant features and base-10 organization of the game board measured by the game board reproduction task. The mean was $4.62(S D=2.73)$ on the pretest and $4.38(S D=$ 2.67 ) on the posttest, $t(20)=0.45, p=.66, d=0.09$. This was the anticipated pattern, because experience with the game board was identical for children in Experiment 2 and children in the countfrom- 1 condition of Experiment 1.

\section{Discussion}

The present findings demonstrate that benefits of playing numerical board games are not limited to preschoolers, to the numerical range 1-10, or to games with a strictly linear organization. Substantial learning was observed in kindergartners playing a $0-100$ number board game organized as a semilinear $10 \times 10$ matrix. The findings also indicated that the most rapid and substantial improvement from playing board games occurs during the first two sessions playing the game.

In addition, the findings suggest that encoding is a key process in generating learning from number board games. These results converge with those of previous research that have demonstrated the importance of encoding for mathematics learning (e.g., McNeil \& Alibali, 2004; Prather \& Alibali, 2011). Experiment 1 demonstrated that a subtle difference between two ways of playing a number board game-whether children counted-from-1 or counted-on from the larger number-had a large impact on learning. Counting-on led to improvements in number line estimation, numeral identification, counting from numbers other than 1 , and encoding of the structure of the game board that were roughly twice as great as the gains of children who counted-from-1 while playing the game. It is especially noteworthy that counting-on led to greater improvements in number line estimation and encoding of positions of numerals on the game board, skills for which counting-on did not provide practice.

Differences between the findings of Experiments 1 and 2 suggest that greater learning of numerical magnitudes occurs when encoding of numbers occurs within the context of a number board game where children can connect the numbers to visual, auditory, kinesthetic, and temporal magnitude cues than when the encoding of numbers occurs outside the game context. Encoding numerals outside of a game context produced improvement in numeral identification and counting from numbers other than 1 but no significant improvement in number line estimation. Thus, gains from counting-on while playing the board game could not be attributed to counting-on from numbers other than 1 or to counting in the presence of printed numbers.

\section{General Discussion}

In this concluding section, we examine several implications of these findings.

\section{The Cognitive Alignment Framework}

The cognitive alignment framework proposes that the more precise the alignment among the desired mental representation, the physical materials being used to promote learning, and the activities that direct learners' thoughts and actions during the acquisition process, the greater the learning is likely to be. Key features of desired mental representations must be instantiated in the physical materials for learning of that representation to occur. The presence in the physical materials of relevant information, however, does not guarantee its encoding. Even simple well-designed physical materials include irrelevant cues and can be used without the desired encoding.

One way to increase encoding of key structural features is to organize activities in ways that promote the desired encoding. The advantage of the count-on condition was predicted, because naming the number in each square requires its encoding, which provides the data needed to correlate numbers in different squares with the visuo-spatial, auditory, kinesthetic, and temporal cues that accompany reaching that square. In contrast, the counting-from-1 procedure does not require, or even encourage, attention to the numbers in the squares. Consistent with this interpretation, the numeral position encoding of children in the count-on condition improved about twice as much as that of children in the countfrom-1 condition. Moreover, results of the mediation analyses indicated that improved encoding in the count-on condition mediated the acquisition of more linear number line estimates. Finally, the results of Experiment 2 indicated that encoding numerals outside of the context of the game did not lead to improvement in number line estimation. This finding provides further evidence for the idea that naming the number in each square facilitated the encoding of the numerical-spatial relations on the game board that offer information about numerical magnitude. The current data did not allow us to determine which cues most facilitated learning about numerical magnitudes, but the data do indicate that the cues to numerical magnitude are insufficient to promote learning without activities that promote encoding of the numbers.

If young children had spontaneously encoded the numbers in the squares, the count-on procedure would have been unnecessary. However, 5- and 6-year-olds do not automatically encode numbers from 1 to 100 in situations that do not demand it (Girelli, Lucangeli, \& Butterworth, 2000; van Galen \& Reitsma, 2008) or when learners have limited numerical knowledge (Barrett et al., 1993; Ornstein et al., 1998). Better numeral identification skills might have reduced the working memory demands of the count-on procedure, thus facilitating formation of a linear representation of the game board. 
These results suggest that additional testing of the cognitive alignment framework would be worthwhile. For example, the framework suggests that to the extent that kinesthetic cues influence learning, having children observe an experimenter moving the token while counting-on should produce less learning than having children move the token while counting-on themselves.

A further prediction of the cognitive alignment framework is that procedures that lead children to note their token's position relative to that of other players' tokens, and relative to the starting and ending points on the board, would facilitate the formation of linear representations of numerical magnitudes. Such procedures could help children learn relations among numbers, just as procedures that encourage structurally relevant comparisons of the properties of objects do in analogical reasoning (Gentner \& Markman, 1997; Namy \& Gentner, 2002). For example, children might be told, "The closer to the goal (i.e., 100) the token is, the greater the number in that square," and "The more squares between one's current position and that of an opponent, the greater the difference between the numbers on which the tokens sit." If these predictions prove accurate, they will illustrate the usefulness of the cognitive alignment framework, in particular, and of developmental psychology theory, in general, for understanding and designing educational interventions.

\section{Effects of Spatial Organization of Physical Materials}

Not all spatial arrangements of numbers within board games produce comparable learning. Siegler and Ramani (2009) found that preschoolers learned little about numerical magnitudes from playing a $0-10$ number board game when the 10 numbers were displayed in a circular pattern. The current data provide evidence that playing a $0-100$ number board game with the numbers organized semilinearly in a $10 \times 10$ grid akin to that used in Chutes and Ladders improves kindergartners' knowledge of numerical magnitudes, counting-on, and numeral identification. These results indicate that a game board does not need to be strictly linear for acquisition of a linear representation of numbers in that range to be acquired.

One possible explanation of the difference between the lack of learning with the circular arrangement and the substantial learning from the $10 \times 10$ matrix is that the matrix was easier to transform into a strictly linear array. Another interpretation was that the circular organization of the boards in Siegler and Ramani (2009) interfered with a nascent linear organization that the preschoolers had begun to form but that was not too weak to yield good outcomes on magnitude comparison and number line estimation tasks. A third interpretation was that the older children (kindergartners rather than preschoolers) in this study were better able to make the transformation needed to represent the $10 \times 10$ matrix as a strictly linear array.

A fourth interpretation, and in many ways the most intriguing, is that the $10 \times 10$ matrix might have helped children learn the base-10 structure of the number system, which, in turn, might be important for understanding numerical magnitude of numbers greater than 10 . Within the present board game, the rows reflected the decade structure and the columns the unit structure of the base-10 system ( 1 was in the leftmost bottom square, 11 directly above 1, 21 directly above 11, etc.) This organization differs from that of Chutes and Ladders, which snakes around with 11 directly above 10 and 21 directly above 20 . We adopted the present organization because it seemed more likely to convey to children the base-10 structure of the number system. The improved performance on the encoding tasks shown by all the children, but especially by those in the count-on condition, suggests that this goal was realized. It was of course possible that children might have remembered the exact location of individual numbers without abstracting the game's structure, but observations of children counting the decades (" $10,20,30 ")$ and then the units (" 31,32 , 33 ), together with the difficulty of remembering 100 locations after only eight exposures to each, made this unlikely.

These findings raise the question of whether the present organization is more useful for abstracting the structure of the base-10 system than the traditional organization of the Chutes and Ladders board. If the present organization proves to promote greater learning of the base-10 system, toy companies that produce games such as Chutes and Ladders might want to incorporate this design feature into future versions of the game.

\section{Limitations}

Board games can increase children's numerical knowledge, but their effects may vary with children's engagement with the games. Such engagement was not measured in the present study, but it is possible that the count-on condition was more engaging than the count-from- 1 condition because it was more challenging. Alternatively, it is possible that the count-from-1 condition was more engaging because the opportunity to win came at a lower cognitive cost. Future studies could include a larger sample and a measure of individuals' effortful control to test the relation between the type of counting used and engagement in the game. If counting-on was more demanding, children higher in effortful control might benefit more from that condition, because they would be engaged despite its challenges. Effortful control would be expected to matter less for children in the count-from- 1 condition because of that condition's lower cognitive demands. Future studies also could include a measure of engagement to examine which features of games produce high levels of engagement and whether level of engagement influences learning.

Another limitation of the present study is that it did not test the long-term benefits of playing the 0-100 game. Ramani and Siegler (2008) demonstrated that playing a similar 0-10 linear number board game produced gains in mathematical knowledge that endured for at least 2 months. Including delayed posttests in a future experiment would allow examination of how long benefits of more complex board games endure.

\section{Conclusion}

Identifying general principles that predict when and explain how games produce learning is a worthy goal of future research. Over $\$ 2$ billion a year is spent on games and puzzles in the United States (Toy Industry Association, 2012). Many of these games are used by parents and teachers as tools for inculcating knowledge of colors, numbers, letters, and words, as well as a wide range of facts, concepts, modes of reasoning, and problem-solving techniques. Research on what and how children learn from such games can suggest simple, inexpensive ways to modify the games to increase learning. The present study provides one example of how this can be done. 


\section{References}

Ainley, J. (1990). Playing games and learning mathematics. In L. P. Steffe \& T. Wood (Eds.), Transforming children's mathematics education: International perspectives (pp. 84-91). Hillsdale, NJ: Erlbaum.

Alibali, M. W. (1999). How children change their minds: Strategy change can be gradual or abrupt. Developmental Psychology, 35, 127-145. doi:10.1037/0012-1649.35.1.127

Aunola, K., Leskinen, E., Lerkkanen, M. K., \& Nurmi, J. E. (2004). Developmental dynamics of math performance from preschool to Grade 2. Journal of Educational Psychology, 96, 699-713. doi:10.1037/00220663.96.4.699

Ball, D. L. (1992). Magical hopes: Manipulatives and the reform of math education. American Educator, 16, 14-18.

Baron, R. M., \& Kenny, D. A. (1986). The moderator-mediator variable distinction in social psychological research: Conceptual, strategic, and statistical considerations. Journal of Personality and Social Psychology, 51, 1173-1182. doi:10.1037/0022-3514.51.6.1173

Barrett, S. E., Abdi, H., Murphy, G. L., \& Gallagher, J. M. (1993). Theory-based correlations and their role in children's concepts. Child Development, 64, 1595-1616. doi:10.2307/1131458

Berch, D. B., Foley, E. J., Hill, R. J., \& McDonough-Ryan, P. M. (1999). Extracting parity and magnitude from Arabic numerals: Developmental changes in number processing and mental representation. Journal of Experimental Child Psychology, 74, 286-308. doi:10.1006/jecp.1999 .2518

Blaxton, T. A. (1989). Investigating dissociations among memory measures: Support for a transfer-appropriate processing framework. Journal of Experimental Psychology: Learning, Memory, and Cognition, 15, 657-668. doi:10.1037/0278-7393.15.4.657

Booth, J. L., \& Siegler, R. S. (2006). Developmental and individual differences in pure numerical estimation. Developmental Psychology, 42, 189-201. doi:10.1037/0012-1649.41.6.189

Booth, J. L., \& Siegler, R. S. (2008). Numerical magnitude representations influence arithmetic learning. Child Development, 79, 1016-1031. doi: 10.1111/j.1467-8624.2008.01173.x

Brown, A. L., Kane, M. J., \& Echols, K. (1986). Young children's mental models determine analogical transfer across problems with a common goal structure. Cognitive Development, 1, 103-121. doi:10.1016/S08852014(86)80014-4

Bugden, S., \& Ansari, D. (2011). Individual differences in children's mathematical competence are related to the intentional but not automatic processing of Arabic numerals. Cognition, 118, 32-44. doi:10.1016/j .cognition.2010.09.005

Chen, Z. (1996). Children's analogical problem solving: The effects of superficial, structural, and procedural similarity. Journal of Experimental Child Psychology, 62, 410-431. doi:10.1006/jecp.1996.0037

Chi, M. T. H. (1978). Knowledge structures and memory development. In R. S. Siegler (Ed.), Children's thinking: What develops? (pp. 73-96). Hillsdale, NJ: Erlbaum.

DeLoache, J. S., Kolstad, V., \& Anderson, K. (1991). Physical similarity and young children's understanding of scale models. Child Development, 62, 111-126. doi:10.2307/1130708

Din, F. S., \& Calao, J. (2001). The effects of playing educational video games on kindergarten achievement. Child Study Journal, 31, 95-102.

Duncan, G. J., Dowsett, C. J., Claessens, A., Magnuson, K., Huston, A. C., Klebanov, P., . . . Japel, C. (2007). School readiness and later achievement. Developmental Psychology, 43, 1428-1446. doi:10.1037/00121649.43.6.1428

Geary, D. C. (2011). Cognitive predictors of achievement growth in mathematics: A 5-year longitudinal study. Developmental Psychology, 47, 1539-1552. doi:10.1037/a0025510

Geary, D. C., Hoard, M. K., Byrd-Craven, J., Nugent, L., \& Numtee, C. (2007). Cognitive mechanisms underlying achievement deficits in chil- dren with mathematical learning disability. Child Development, 78 , 1343-1359. doi:10.1111/j.1467-8624.2007.01069.x

Gentner, D., \& Markman, A. B. (1997). Structure mapping in analogy and similarity. American Psychologist, 52, 45-56. doi:10.1037/0003-066X .52 .1 .45

Girelli, L., Lucangeli, D., \& Butterworth, B. (2000). The development of automaticity in accessing number magnitude. Journal of Experimental Child Psychology, 76, 104-122. doi:10.1006/jecp.2000.2564

Goswami, U. (1996). Analogical reasoning and cognitive development. In H. Reese (Ed.), Advances in child development and behavior (Vol. 26, pp. 91-138). New York, NY: Academic Press. doi:10.1016/S00652407(08)60507-8

Holloway, I. D., \& Ansari, D. (2009). Mapping numerical magnitudes onto symbols: The numerical distance effect and individual differences in children's math achievement. Journal of Experimental Child Psychology, 103, 17-29. doi:10.1016/j.jecp.2008.04.001

Jordan, N. C., Kaplan, D., Ramineni, C., \& Locuniak, M. N. (2009). Early math matters: Kindergarten number competence and later mathematics outcomes. Developmental Psychology, 45, 850-867. doi:10.1037/ a0014939

Laski, E. V., \& Siegler, R. S. (2007). Is 27 a big number? Correlational and causal connections among numerical categorization, number line estimation, and numerical magnitude comparison. Child Development, 78, 1723-1743. doi:10.1111/j.1467-8624.2007.01087.x

LeFevre, J.-A., Fast, L., Skwarchuk, S.-L., Smith-Chant, B. L., Bisanz, J., Kamawar, D., \& Penner-Wilger, M. (2010). Pathways to mathematics: Longitudinal predictors of performance. Child Development, 81, 17531767. doi:10.1111/j.1467-8624.2010.01508.x

McCloskey, M., \& Kaiser, M. (1984). The impetus impulse: A medieval theory of motion lives on in the minds of children. Sciences, 24, 40-46. doi:10.1002/j.2326-1951.1984.tb02754.x

McNeil, N. M., \& Alibali, M. W. (2004). You'll see what you mean: Students encode equations based on their knowledge of arithmetic Cognitive Science, 28, 451-466. doi:10.1016/j.cogsci.2003.11.002

McNeil, N., \& Jarvin, L. (2007). When theories don't add up: Disentangling the manipulatives debate. Theory Into Practice, 46, 309-316. doi:10.1080/00405840701593899

Namy, L. L., \& Gentner, D. (2002). Making a silk purse out of two sow's ears: Young children's use of comparison in category learning. Journal of Experimental Psychology: General, 131, 5-15. doi:10.1037/00963445.131.1.5

Ornstein, P. A., Merritt, K. A., Baker-Ward, L., Furtado, E., Gordon, B. N., \& Principe, G. (1998). Children's knowledge, expectation, and longterm retention. Applied Cognitive Psychology, 12, 387-405. doi: 10.1002/(SICI)1099-0720(199808)12:4<387::AID-ACP574>3.0.CO;2-5

Prather, R., \& Alibali, M. W. (2011). Children's acquisition of arithmetic principles: The role of experience. Journal of Cognition and Development, 12, 332-354. doi:10.1080/15248372.2010.542214

Preacher, K. J., \& Hayes, A. F. (2008). Asymptotic and resampling strategies for assessing and comparing indirect effects in multiple mediator models. Behavior Research Methods, 40, 879-891. doi:10.3758/ BRM.40.3.879

Ramani, G. B., \& Siegler, R. S. (2008). Promoting broad and stable improvements in low-income children's numerical knowledge through playing number board games. Child Development, 79, 375-394. doi: 10.1111/j.1467-8624.2007.01131.x

Ramani, G. B., \& Siegler, R. S. (2011). Reducing the gap in numerical knowledge between low- and middle-income preschoolers. Journal of Applied Developmental Psychology, 32, 146-159. doi:10.1016/j.appdev 2011.02.005

Schneider, M., Grabner, R. H., \& Paetsch, J. (2009). Mental number line, number line estimation, and mathematical school achievement: Their interrelations in Grades 5 and 6. Journal of Educational Psychology, 101, 359-372. doi:10.1037/a0013840 
Siegler, R. S. (1976). Three aspects of cognitive development. Cognitive Psychology, 8, 481-520. doi:10.1016/0010-0285(76)90016-5

Siegler, R. S. (2006). Microgenetic analyses of learning. In W. Damon \& R. M. Lerner (Series Eds.) \& D. Kuhn \& R. S. Siegler (Vol. Eds.), Handbook of child psychology: Vol. 2. Cognition, perception, and language (6th ed., pp. 464-510). Hoboken, NJ: Wiley. doi:10.1002/ 9780470147658.chpsy0211

Siegler, R. S., \& Booth, J. (2004). Development of numerical estimation in young children. Child Development, 75, 428-444. doi:10.1111/j.14678624.2004.00684.x

Siegler, R. S., \& Chen, Z. (1998). Developmental differences in rule learning: A microgenetic analysis. Cognitive Psychology, 36, 273-310. doi:10.1006/cogp.1998.0686

Siegler, R. S., \& Ramani, G. B. (2008). Playing board games promotes low-income children's numerical development. Developmental Science, 11, 655-661. doi:10.1111/j.1467-7687.2008.00714.x

Siegler, R. S., \& Ramani, G. B. (2009). Playing linear number board games-but not circular ones-improves low-income preschoolers' numerical understanding. Journal of Educational Psychology, 101, 545560. doi:10.1037/a0014239

Siegler, R. S., Thompson, C. A., \& Schneider, M. (2011). An integrated theory of whole number and fractions development. Cognitive Psychology, 62, 273-296. doi:10.1016/j.cogpsych.2011.03.001

Staszewski, J. J. (1988). Skilled memory and expert mental calculation. In
M. Chi, R. Glaser, \& M. J. Farr (Eds.), The nature of expertise (pp. 71-128). Hillsdale, NJ: Erlbaum.

Stevenson, H. W., \& Newman, R. S. (1986). Long-term prediction of achievement and attitudes in mathematics and reading. Child Development, 57, 646-659. doi:10.2307/1130343

Toy Industry Association. (2012). Annual U.S. domestic toy sales data. Retrieved July 3,2013, from http://www.toyassociation.org/App_Themes/ tia/pdfs/facts/RollingData.pdf

Uttal, D. H., O’Doherty, K., Newland, R., Hand, L. L., \& DeLoache, J. (2009). Dual representation and the linking of concrete and symbolic representations. Child Development Perspectives, 3, 156-159. doi: 10.1111/j.1750-8606.2009.00097.x

van Galen, M. S., \& Reitsma, P. (2008). Developing access to number magnitude: A study of the SNARC effect in 7- to 9- year-olds. Journal of Experimental Child Psychology, 101, 99-113. doi:10.1016/j.jecp .2008.05.001

Whyte, J. C., \& Bull, R. (2008). Number games, magnitude representation, and basic number skills in preschoolers. Developmental Psychology, 44, 588-596. doi:10.1037/0012-1649.44.2.588

Received August 2, 2012

Revision received July 24, 2013

Accepted July 30, 2013 